\title{
THE SOLUTION AND THE STABILITY OF A NONLINEAR AGE-STRUCTURED POPULATION MODEL
}

\author{
NORHAYAT ${ }^{1}$ and G. C. WAKE ${ }^{2}$ \\ (Received 7 September, 2001; revised 6 August, 2002)
}

\begin{abstract}
We consider an age-structured population model achieved by modifying the classical Sharpe-Lotka-McKendrick model, incorporating an overcrowding effect or competition for resources term. This term depends on the whole population rather than on any specific age group, in the case of overcrowding or limitation of resources. We investigate the solutions for arbitrary initial conditions. We consider the existence of a steady age distribution and its stability and are able to determine this for a simple illustrative case. If the non-trivial steady age distribution is unstable, there is a critical initial population size beyond which the population explodes. This watershed is independent of the shape of the initial age distribution.
\end{abstract}

\section{Introduction}

Some of the earliest population models developed were proposed by Malthus in 1798 [6] and then by Verhulst in 1838. These models are unstructured and so ignore the properties of individuals and in particular ageing of individuals is not taken into account. Hence they consider only the total number of individuals in the population. The limitation of these models is that all members of the population are assumed to be equally likely to die or to reproduce. However, the Verhulst population model allows for overcrowding effects and limitation of resources, and the population approaches a non-trivial equilibrium state called the carrying capacity $K$ as time, $t$, approaches infinity. In other words, the rate of growth becomes depressed as the total population increases, to compensate for the effect of overcrowding until it reaches the carrying capacity which is an equilibrium state.

\footnotetext{
${ }^{1}$ Department of Mathematics, University Brunei Darussalem, Negara Brunei Darussalem; e-mail: yati@fos.ubd.edu.bn.

${ }^{2}$ Department of Mathematics and Statistics, University of Canterbury, Private Bag 4800, Christchurch, New Zealand; e-mail: g.wake@math.canterbury.ac.nz.

(C) Australian Mathematical Society 2003, Serial-fee code 1446-1811/03
} 
It is only relatively recently that the effect of age of the individuals in a population on the growth of that population has been considered. Among the first 'continuous' population models incorporating age effects were those of Sharpe and Lotka [10] and McKendrick [7]. Basically, the Sharpe-Lotka-McKendrick model assumes that birth and mortality processes are linear functions of population density. Leslie [4] used discrete age compartments (giving rise to Leslie matrices) and in [3] used both discrete time and age compartments. The methods used here generalise these for a completely non-discrete model introduced below. Gurtin and MacCamy [1] and Hoppensteadt [2] introduced the first models of nonlinear continuous age-dependent population dynamics. Wake et al. [11] considered age-structured disease models but with nonlinear terms in the disease-transmission formulation. Here we consider the effect of overall population density on the age distribution as suggested by Pollak [9]. Consequently, the equations of that model, as in the case of the Verhulstian models, contained nonlinear terms involving the total population. By analogy with the Verhulstian models of age-independent population dynamics, these nonlinearities provided a mechanism by which the population might stabilise to a non-trivial equilibrium state as time evolves.

\section{The formulation of the age-structured model}

The classical model of linear age-dependent population dynamics represented by the Sharpe-Lotka equation is formulated as follows.

Let $n(a, t)$ be the population density (or age distribution) with respect to age $a$ of a population at time $t$. The units of $n(a, t)$ are given in units of population divided by units of time. Accordingly, the total number of individuals between ages $a_{1}$ and $a_{2}$ is given by

$$
\int_{a_{2}}^{a_{1}} n(a, t) d a,
$$

where $n(a, t)$ is taken to be a smooth function of $(a, t)$. The total population at time $t$ of all members of the population is given by

$$
N(t)=\int_{0}^{\infty} n(a, t) d a .
$$

The upper limit of the chronological age of the species considered is, of course, some finite number, but it will be assumed for convenience to be infinite with $n(\infty, t)=0$ and with $N(t)$ being finite. This density function satisfies the so-called balance law (or ageing process of the population).

The equation describing the growth of the population is formulated as follows. 
The change occurring in the population of individuals of age $a$ at a time $t$ in a particular time interval is proportional to the product of the size of the population and the length of time interval $d t$. (Note that the small time interval of size $d t$ may be chosen for convenience as equal to $d a$ as used by Nisbet and Gurney [8].)

Thus we have change in $n=$ rate of removal by death over time interval $d t$, or

$$
n(a+d a, t+d t)-n(a, t)=-\mu(a, t) n(a, t) d t
$$

where $\mu(a, t)$ is the nonnegative age-dependent death rate. By dividing this equation by $d t$ and finding the limit as $d t \rightarrow 0$ ( $d t=d a$, of course), we have

$$
\frac{\partial n}{\partial t}+\frac{\partial n}{\partial a}=-\mu(a, t) n, \quad t, a>0 .
$$

This is the equation for age-dependent population growth which is a linear first-order hyperbolic partial differential equation. It is known as the McKendrick equation and derived in other contexts as Von Foerster's equation. Here only the death rate (removal) is included and the birth rate is not involved in the equation because, when individuals are born at $a=0$, they are introduced into the population through the boundary (renewal) condition.

The number of newborn individuals introduced into the population is given by

$$
n(0, t)=\int_{0}^{\infty} \beta(a) n(a, t) d a,
$$

where $\beta$ gives the nonnegative age-dependent birth rate such that $\beta(a) d a$ is the average number of offspring produced per unit time by an individual of age $a$ in time interval $(t, t+d t)$. The above equation is also called the renewal equation. The value of $n(0, t)$ at any time $t$ depends on the age distribution of the population at that time, as determined by the integral of the density $n(a, t)$ weighted with the specific birth rate $\beta(a)$.

Finally, the initial age-distribution of the population is assumed to be known

$$
n(a, 0)=n_{0}(a)
$$

This is a known nonnegative function of age $a$. Notice that (2.2) is not required to hold at $t=0$. If (2.2) does hold at $t=0$, then (2.2) and (2.3) must be compatible in the sense that

$$
\int_{0}^{\infty} \beta(a) n_{0}(a) d a=n_{0}(0) .
$$

The above condition is called the compatibility condition, and is not, in general, required of the initial age distribution. 


\section{The nonlinear age-structured population model}

We now extend our model (2.1) to become nonlinear:

$$
\frac{\partial n}{\partial t}+\frac{\partial n}{\partial a}=-\mu n(1+k N), \quad t, a>0
$$

with renewal condition

$$
n(0, t)=\beta \int_{0}^{\infty} n(a, t) d a,
$$

where we have assumed, for simplicity, that $\beta$ and $\mu$ are independent of age, and initial age distribution

$$
n(a, 0)=n_{0}(a)
$$

The assumption of constant birth rate and the specific non-age-dependence of the death rate is made so that the existence (under certain conditions) of a supercritical bifurcation can be obtained analytically. Also the nature of the watershed for the subcritical bifurcation (when it exists) can be easily quantified. The model here is genuinely age-structured as seen by the solution given below (see (4.4)) where the steady-age distribution is non-constant (see (5.5)).

We also define the total population at time $t$ introduced in (3.1) to be

$$
N(t)=\int_{0}^{\infty} n(a, t) d a .
$$

The factor $(1+k N)$ on the right-hand side of (3.1) contributes to limiting effects which may be due to overcrowding effects and lack of resources. As such it is not dependent on a "local age group" but on the whole population. The parameter $k$ can either be positive or negative. The former case $(k>0)$ is the most familiar: "over-crowding", the effects of increased disease, competition etc. causing an increased death rate. The latter case $(k<0)$ is less familiar in practice, but does arise in less sophisticated population cohorts, where predation rates etc. decrease with population size $N$.

\section{The solutions of the nonlinear age-structured model}

The solutions of our model for a general arbitrary function $n_{0}(a)$ may be found by taking the Laplace transform in $a$ for $n(a, t)$, which is given by

$$
\bar{n}(p, t)=\int_{0}^{\infty} e^{-p a} n(a, t) d a .
$$


Then our model becomes

$$
\frac{\partial \bar{n}}{\partial t}+p \bar{n}-n(0, t)=-\mu \bar{n}(1+k N(t)) .
$$

But by (3.2) and (3.4), we have $n(0, t)=\beta N(t)$. Hence we have to solve the equation

$$
\frac{\partial \bar{n}}{\partial t}+[p+\mu+\mu k N(t)] \bar{n}=\beta N(t)
$$

with initial condition $\bar{n}(p, 0)=\bar{n}_{0}(p)$.

Therefore we find $\bar{n}(p, t)$ to be

$$
\bar{n}(p, t)=W \exp \left(-p t-\mu t-\mu k \int_{0}^{t} N(s) d s\right),
$$

where $W=\beta \int_{0}^{t} N(r) e^{p r+\mu k \int_{0}^{\prime} N(s) d s} d r+\bar{n}_{0}(p)$.

The population density can then be found by inverting the above equation. We thus obtain

$$
\begin{aligned}
n(a, t)= & \beta e^{-\mu a} e^{-\mu k \int_{t-a}^{t} N(s) d s} N(t-a) H(t-a) \\
& +n_{0}(a-t) H(a-t) e^{-\mu k \int_{0}^{\prime} N(s) d s},
\end{aligned}
$$

where $H$ is the Heaviside (unit step) function.

Equation (4.2) satisfies the equation $n(0, t)=\beta N(t)$ trivially. From (3.4) we have that

$$
N(t)=\int_{0}^{\infty} n(a, t) d a=\int_{0}^{t} n(a, t) d a+\int_{1}^{\infty} n(a, t) d a .
$$

Therefore substituting (4.2) into the above equation and evaluating the integrals, we obtain the total population $N(t)$ :

$$
N(t)=\frac{(\beta-\mu) N_{0}}{\left[\beta-\mu-\mu k N_{0}\right] e^{(\mu-\beta) t}+\mu k N_{0}}
$$

such that $\int_{t}^{\infty} n_{0}(a-t) d a=\int_{0}^{\infty} n_{0}(r) d r \equiv N_{0}$, where $N(0)=N_{0}$ is the total number of individuals alive at $t=0$.

It is possible to substitute (4.3) into the solution (4.2) to get

$$
n(a, t)= \begin{cases}\frac{\beta(\beta-\mu) N_{0} e^{(\beta-\mu)(t-a)}}{\left(\beta-\mu-\mu k N_{0}\right)+\mu k N_{0} e^{(\beta-\mu) t}}, & t>a \\ \frac{\beta(\beta-\mu) n_{0}(a-t)}{\left(\beta-\mu-\mu k N_{0}\right)+\mu k N_{0} e^{(\beta-\mu) t}}, & t<a .\end{cases}
$$

Of course, we could obtain (2.1) by solving the integrated form of (3.1); but we could not obtain $n(a, t)$ this way, nor fully investigate the dependence of the stability of any s.a.d. on the initial condition $n_{0}(a)$, which we discuss in the next section. 


\section{The steady age distribution and its stability}

For our simple model described in Section 3, we are particularly interested in the long-term behaviour or steady age distribution (s.a.d.). As such, before we begin our investigation, we assume that there exists a critical value $C$, say $C_{\mathrm{cr}}$. This is said to represent the edge of the stable manifold of $n_{s}(a) \equiv 0$.

Let us first define an equilibrium solution to (3.1)-(3.3), $n(a, t) \rightarrow n_{s}(a)$, that is independent of time. The function $n_{s}(a)$ is the s.a.d.

An equilibrium solution will satisfy the equation

$$
\frac{\partial n_{s}}{\partial a}=-\mu n_{s}\left(1+k N_{s}\right)
$$

and the renewal condition from (3.2) is then simplified to become

$$
n_{s}(0)=\beta \int_{0}^{\infty} n_{s}(a) d a=\beta N_{s},
$$

where $n_{s}(0)$ is the (constant) birth rate at equilibrium. The solution of the above equation then becomes

$$
n_{s}(a)=L \exp \left[-\mu a\left(1+k N_{s}\right)\right]
$$

where $n_{s}(0)=L$.

Substituting (5.1) into the renewal condition, we obtain

$$
1=\frac{\beta}{\left(1+k N_{s}\right)} .
$$

We denote this as $R(0)$, representing the expected number of offspring produced in a lifetime by an individual in the absence of disease and set it equal to 1. Equation (5.2) is required for the existence of an s.a.d. and only then can this case be examined further. (This is particularly useful in determining the stability and will be discussed in Section 6.)

Rearranging (5.1) we have

$$
N_{s}=\frac{\beta-\mu}{\mu k} .
$$

From (3.4), the total population is $N_{s}=\int_{0}^{\infty} n_{s}(a) d a$. Substituting (5.1) into the above equation gives

$$
N_{s}=\frac{L}{\mu\left(1+k N_{s}\right)} .
$$


To find the value for the arbitrary constant $L$, we substitute (5.3) into this expression to give

$$
L=\mu\left(N_{s}+k N_{s}^{2}\right)=\mu\left(\frac{\beta-\mu}{\mu k}+k\left(\frac{\beta-\mu}{\mu k}\right)^{2}\right) .
$$

Simplifying further, we have

$$
L=\frac{\beta(\beta-\mu)}{\mu k} .
$$

Combining (4.4), (5.2) and (5.3), we obtain

$$
n_{s}(a)=\frac{\beta(\beta-\mu)}{\mu k} e^{-\beta a} \quad \text { and } \quad N_{s}=\frac{\beta-\mu}{\mu k} .
$$

This is the equation of the non-trivial s.a.d. for our model.

\section{General discussion on the stability of the steady-state distribution}

We shall consider the simplest and most common notion of (Lyapunov) stability for differential equation systems. Our approach will be the standard method of linearising the model about a solution (usually an equilibrium) whose stability or instability is of interest. We have denoted the equilibrium solution to be $n_{s}(a)$. This method of course yields stability results which are in general only local near the solution being considered. Although such local stability results are sometimes criticised as being too restrictive and inappropriate in general for the study of what should be a broader concept of 'ecological stability', we will justify this approach by saying that such a study contributes at least a first step towards understanding the qualitative nature of the models considered here. Further, the outcome should reflect the global results, which we obtain in full later.

Let us first of all assume that the solution to our model is of the form

$$
n(a, t)=n_{s}(a)+u(a, t) .
$$

Substituting this into (3.1) and linearising the resulting equations about $u=0$ gives

$$
\frac{\partial u}{\partial t}+\frac{\partial u}{\partial a}=-\mu k n_{s}(a) \int_{0}^{\infty} u(a, t) d a .
$$

We try a solution of the form $u(a, t)=g(a) e^{\lambda t}$, where $\lambda$ is a constant. Substituting this into (6.1) and simplifying gives

$$
g^{\prime}(a)=-(\mu+\lambda) g(a)-\mu k n_{s}(a) \int_{0}^{\infty} g(a) d a .
$$


Solving this and using the renewal equation which is now

$$
g(0)=\beta \int_{0}^{\infty} g(a) d a
$$

we get

$$
g(a)=A\left(e^{-(\mu+\lambda) a}-\frac{\mu k}{\beta} \int_{0}^{a} e^{-(\mu+\lambda)(a-b)} n_{s}(b) d b\right),
$$

where $g(0)=A$ is a constant. Putting this last expression in the renewal condition (6.3) we get, for $A \neq 0$,

$$
1=R(\lambda) \equiv \frac{\beta}{\mu+\lambda}-\mu \int_{0}^{\infty} \int_{0}^{a} e^{-(\mu+\lambda)(a-b)} n_{s}(b) d b d a .
$$

The equation of this form is often known as the characteristic equation. This type of equation was first discovered by Lotka [6]. Here the birth rate $\beta$ and death rate parameter $\mu$ are taken to be constants. The right-hand side of (6.4) is a strictly decreasing function of $\lambda$ which assumes all values in $(-\infty, \infty)$. In this case, (6.4) has a unique real solution $\lambda^{*}$ and for this choice of $\lambda^{*},(6.4)$ describes the stability of the s.a.d. for all values of $A$ or $g(0)$ depending upon the sign of $\lambda^{*}$. Substituting the two expressions for $n_{s}(a)$ into (6.4) will determine the local stability of these s.a.d.'s. However, this is more easily determined by the exact solution in (4.4) for $n_{s}(a) \neq 0$.

\section{The analysis of the stability of the s.a.d.'s}

In Section 4, we have found the expression for the total population to be given by (4.3). We also found the expression for $n(a, t)$ in (4.4). Let us now look at the stability of the s.a.d. of our model at the following intervals

(A) the trivial solution $(N(t) \neq 0)$,

(B) the two non-trivial branches. The two branches are:

- when $\beta>\mu$ and $k>0$ and

- when $\beta<\mu$ and $k<0$.

Case A. For the trivial solution, first of all we have that (5.2) takes the form $R(0)=1=\beta / \mu$ and (6.4) takes the form $R(0)=\beta /(\mu+\lambda)$.

However, $R(\lambda)$ is a decreasing function of $\lambda$, so that if $R(0)<1$ we have $\lambda<0$ and the trivial solution is asymptotically stable. For $\beta>\mu$, in contrast we have $\lambda>0$ and the trivial solution is unstable.

Case B. (i) If $\beta>\mu$ and $k>0$, then the term in the denominator of (4.3) which dominates at large $t$ is $\mu k N_{0}$ so that $N(t) \rightarrow(\beta-\mu) / \mu k$. This means that on 


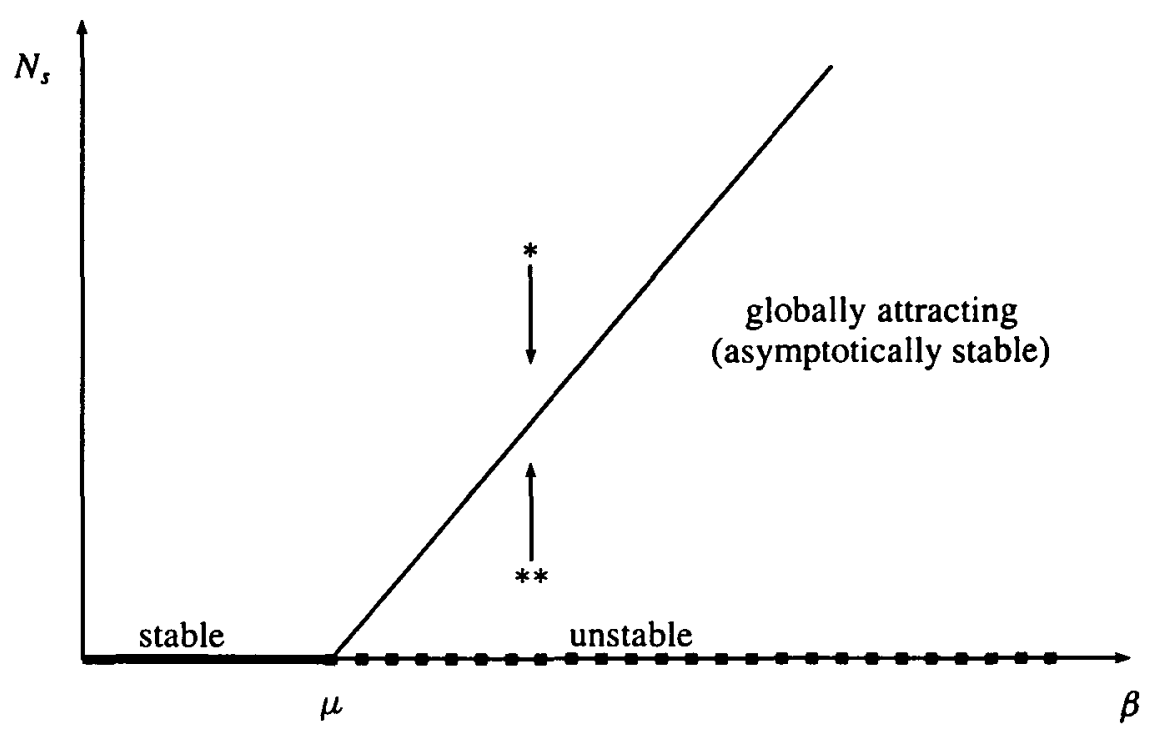

FIGURE l. The sketch of the bifurcation diagram $N_{s}$ against $\beta$ when $k>0$.

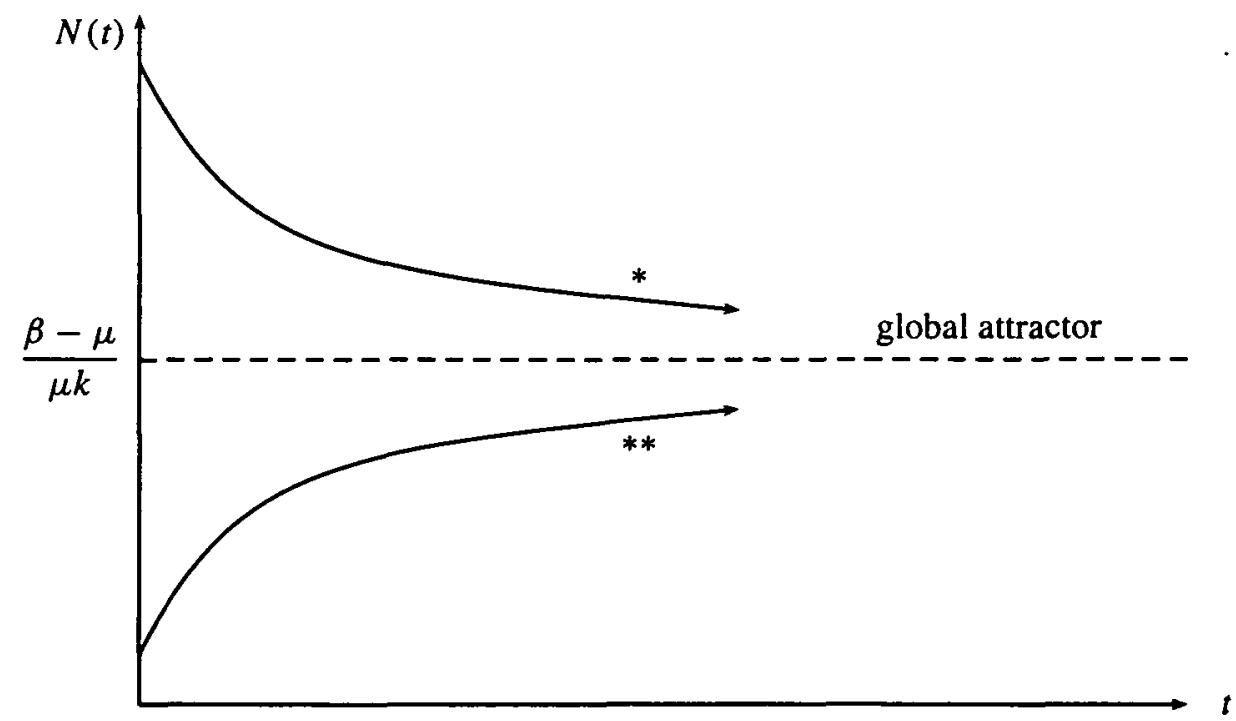

FIGURE 2. The population-time graph (transient diagram) when $\beta>\mu$ and $k>0$ showing the global attractor. 
this branch, $N(t)$ will always tend to the value $(\beta-\mu) / \mu k$. This expression is said to be the global attractor and means that the total population is asymptotically stable and does not depend on the total population size. This is a robust outcome. The bifurcation diagram (Figure 1) and transient diagram (Figure 2) still apply for this problem. Clearly (4.4) shows that $n_{s}(a)$ is also globally asymptotically stable independent of $n_{0}(a)$.

(ii) For $\beta<\mu$ and $k<0,(4.3)$ can be simplified to

$$
N(t)=\frac{1}{\left[\frac{1}{N_{0}}+\frac{\mu k}{\mu-\beta}\right] e^{(\mu-\beta) t}-\frac{\mu k}{\mu-\beta}} .
$$

We shall now consider the following cases in conjunction with the above equation.

Case 1. $N(t) \rightarrow 0$ as $t \rightarrow \infty$ (that is, when $N_{0}$ is sufficiently small); for $N(t) \rightarrow 0$, we need the denominator to tend to infinity. When $t \rightarrow K$ then $e^{(\mu-\beta) t} \rightarrow+K$, but its sign is determined by $k$. For it to be positive we require

$$
\left[\frac{1}{N_{0}}+\frac{\mu k}{\mu-\beta}\right]>0 \text {. }
$$

Therefore we obtain the critical value for the initial total population, $N_{0, \text { cr }}$ given by

$$
N_{0, \text { cr }}=\frac{\beta-\mu}{\mu k}, \quad \text { that is, if } N_{0}<N_{0, \mathrm{cr}}, \quad N(t) \rightarrow 0 .
$$

(Remember $k>0$ and $\mu>\beta$ in this case.)

Case 2. There exists $t^{*}$ such that $N(t) \rightarrow \infty$ as $t \rightarrow t^{*}$ for large $N_{0}$; in this case we need the denominator to be zero at $t=t^{*}$,

or

$$
\left[\frac{1}{N_{0}}+\frac{\mu k}{\mu-\beta}\right] e^{(\mu-\beta) t}=\frac{\mu k}{\mu-\beta}
$$

$$
t^{*}=\frac{1}{\mu-\beta} \ln \left\{\frac{\mu k N_{0}}{\mu-\beta+\mu k N_{0}}\right\} \text {. }
$$

From B (ii) this implies that at this branch, if we start with a total population $N(t)$ below the critical value $N_{0, c r}=(\beta-\mu) / \mu K$, the population will die out and if we start with a value of $N(t)$ above this critical value then the population will explode, that is, $N(t)$ becomes unbounded at $t=t^{*}$. This implies that there is a watershed effect at $N_{0, c r}$. Thus it is unstable (see Figure 3). The population-time graph is represented by Figure 4.

We note that the watershed condition for $N_{0}=N_{0, c r}$ is independent of the shape of the initial conditions. This sometimes occurs when there are multiple non-trivial steady states. 


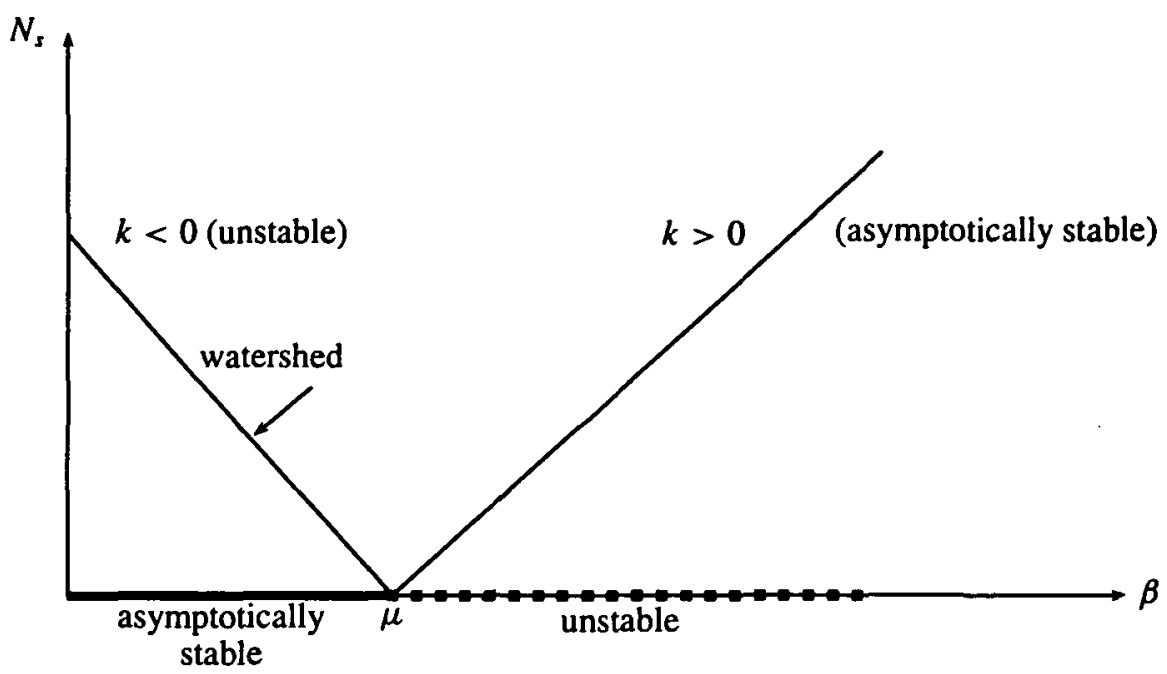

FIGURE 3. The sketch of the bifurcation diagram $N_{s}$ against $\beta$ when $k<0$ showing the watershed at the point $(\beta-\mu) / \mu k$.

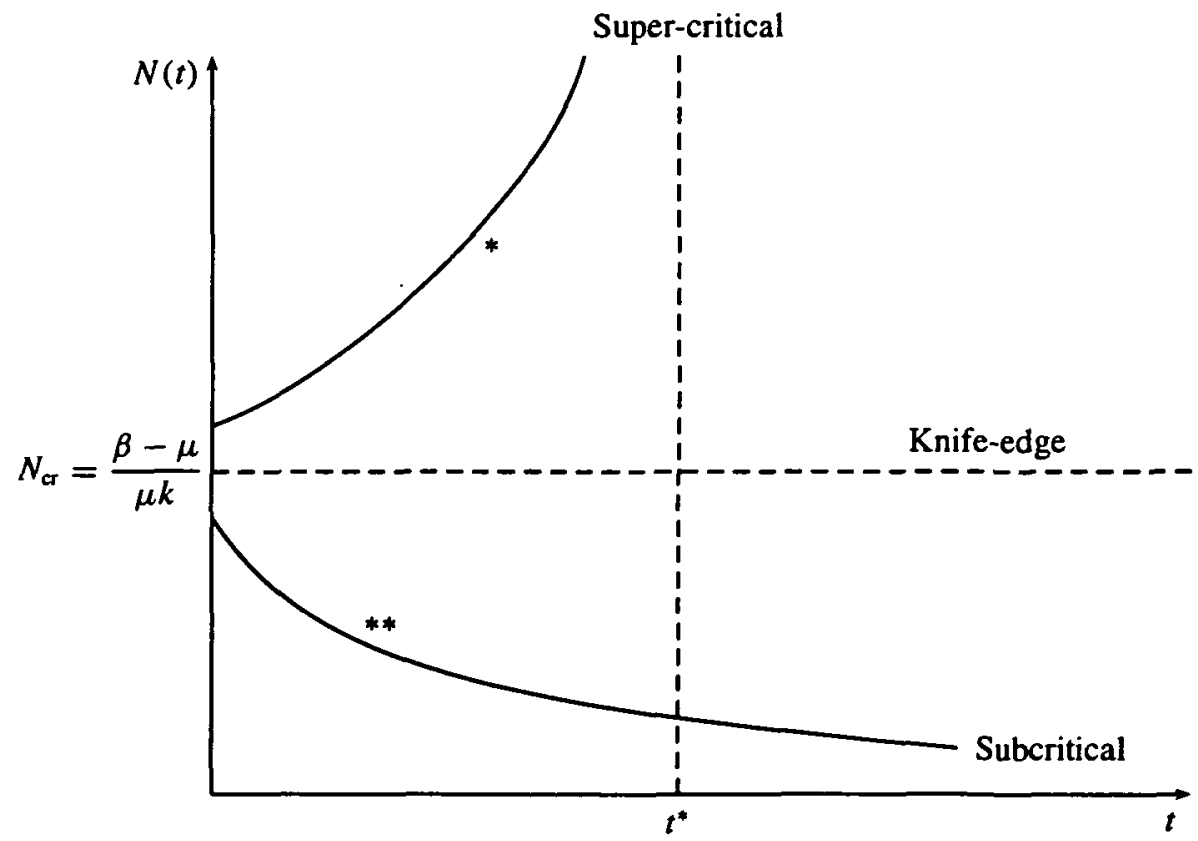

FIGURE 4. The population-time graph (transient diagram) for the case $\beta<\mu$ and $k<0$. 


\section{Conclusions}

We have considered an age-structured population model which includes the effect of overcrowding or limitation of resources affecting the whole population instead of a specific age group only. This is expected to provide a better correlation with real biological situations especially for more highly structured animal populations and better still if we encounter the effect of overcrowding and limitation of resources. Together with the study of a steady age-distribution (s.a.d.) and its stability, we have a better understanding of the long-term behaviour of these solutions. We conclude that there exists a watershed corresponding to an instability if the birth rate is less than the death rate. If the death rate density dependent parameter $k$ is less than zero, there is a critical value of the initial population. This threshold does not depend on the shape of $n_{0}(a)$, only on the total value over all ages, $N_{0}$. This contrasts with what happens in diffusion models. On the other hand, when the birth rate is greater than the death rate there is a global attraction towards the steady-state or survival population.

The next task is to extend this analysis to the even more realistic case of agedependent birth and death processes. Under quite robust and realistic scenarios it is conjectured that the outcomes will be similar.

\section{Acknowledgement}

The authors acknowledge the useful comments of the referees.

\section{References}

[1] M. E. Gurtin and R. C. MacCamy, "Non-linear age-dependent population dynamics", Arch. Ration. Mech. Anal. 5 (1974) 281-300.

[2] F. C. Hoppensteadt, Mathematical theories of populations: demographics, genetics and epidemics (Soc. Industr. Appl. Math., Arrowsmith, England, 1975).

[3] P. H. Leslie, "On the use of matrices in certain population mathematics", Biometrika 33 (1945) 183-212.

[4] P. H. Leslie, "Some further notes on the use of matrices in population mathematics", Biometrika 35 (1949) 213-245.

[5] A. J. Lotka, "The stability of the normal age distribution", Proc. Natl. Acad. Sci. USA 8 (1922) 339-345.

[6] T. R. Malthus, An essay on the principle of population (St. Paul's, London, 1798), reprinted in: T. R. Malthus, An essay on the principle of population and a summary view of the principle of population (Penguin, Harmondsworth, England, 1970).

[7] A. G. McKendrick, "Applications of mathematics to medical problems", Proc. Edinburgh Math. Soc. 44 (1926) 98-130.

[8] R. M. Nisbet and W. S. C. Gurney, Modelling fuctuating populations (Wiley, New York, 1982). 
[9] E. Pollak, "The effective population size of some age-structured populations", Math. Bioscience $168(2000) 36-56$.

[10] F. R. Sharpe and A. J. Lotka, "A problem in age distributions", Phil. Mag. 21 (1911) 435-438.

[11] G. C. Wake, K. Louie and M. G. Roberts, "The regulation of an age-structured population by a fatal disease with or without dispersion", in Proceedings of June $1-4$ of Claremont international conference dedicated to the memory of Stravos Busenberg, (World Scientific, Singapore, 1994) 553-563. 